International Journal of Medical Sciences

ISSN 1449-1907 www.medsci.org 2007 4(5):237-241

Research Paper

CIvyspring International Publisher. All rights reserved

\title{
Theta, alpha and beta burst transcranial magnetic stimulation: brain modulation in tinnitus
}

\author{
Dirk De Ridder 1, Elsa van der Loo 1, Karolien Van der Kelen 1, Tomas Menovsky 1, Paul van de Heyning 1, \\ Aage Moller 2
}

1. Dept of Neurosurgery and ENT, University Hospital Antwerp, Belgium

2. School of Behavioral and Brain Science, University of Texas at Dallas, Dallas, USA

Correspondence to: Dirk De Ridder, Dept of Neurosurgery, University Hospital Antwerp, Wilrijkstraat 10, 2650 Edegem, Belgium. Tel: +32 3 8213336; Fax: +32 3 8252428; dirk.de.ridder@neurosurgery.be

Received: 2007.06.22; Accepted: 2007.10.08; Published: 2007.10.09

Introduction: Some forms of tinnitus are considered to be auditory phantom phenomena related to reorganization and hyperactivity of the auditory central nervous system. Repetitive transcranial magnetic stimulation (rTMS) is a non-invasive tool capable of modulating human brain activity, using single pulse or burst stimuli. Burst rTMS has only been performed in the theta range, and has not been used clinically. The authors analyze whether burst TMS at theta $(5 \mathrm{~Hz})$, alpha $(10 \mathrm{~Hz})$ and beta $(20 \mathrm{~Hz})$ frequencies can temporarily suppress narrow band noise/white noise tinnitus, which has been demonstrated to be intractable to tonic stimulation.

Methods: rTMS is performed both in tonic and burst mode in 46 patients contralateral to the tinnitus side, at 5, 10 and $20 \mathrm{~Hz}$. Fourteen placebo negative rTMS responders are further analyzed.

Results: In 5 patients, maximal tinnitus suppression is obtained with theta, in 2 with alpha and in 7 with beta burst stimulation. Burst rTMS suppresses narrow band/white tinnitus much better than tonic rTMS $t(13)=6.4$, $p<.000$. Women experience greater suppression of their tinnitus with burst stimulation than men, $t(12)=2.9, p<.05$. Furthermore left sided tinnitus is perceived as more distressing on the TQ than right sided tinnitus, $t(12)=3.2$, $\mathrm{p}<.01$. The lower the tinnitus pitch the more effectively rTMS suppresses tinnitus $(r=-0.65, p<0.05)$.

Discussion: Burst rTMS can be used clinically, not only theta burst, but also alpha and beta burst. Burst rTMS is capable of suppressing narrow band/white noise tinnitus very much better than tonic rTMS. This could be due the simple fact that burst neuromodulation is more powerful than tonic neuromodulation or to a differential effect of burst and tonic stimulation on the lemniscal and extralemniscal auditory system. In some patients only alpha or beta burst rTMS is capable of suppressing tinnitus, and theta burst not. Therefore in future rTMS studies it could be worthwhile not to limit burst stimulation to theta burst rTMS.

Key words: burst, neuromodulation, tinnitus, tonic, TMS

\section{Introduction}

Tinnitus is a distressing symptom for which few treatments exist. Some forms of tinnitus are considered to be auditory phantom phenomena [1] similar to central neuropathic pain [2] related to reorganization $[3,4]$ and hyperactivity $[5,6]$ of the auditory central nervous system. Recently it has been shown that stimulation of specific regions of the human brain can alter (suppress) tinnitus in some patients $[7,8]$. Auditory cortex stimulation can be performed with a strong impulse of magnetic field that induces an electrical current in the brain (transcranial magnetic stimulation (TMS)) or with implanted electrodes [7, 8]. Repetitive TMS (rTMS) is a non-invasive tool that makes it possible to selectively and safely stimulate specific regions of the human brain. The electrical current induced in brain tissue by a strong single pulse magnetic field can temporarily excite or inhibit neuronal activity depending on the stimulation parameters [9]. So far it has only been possible to suppress pure tone tinnitus whereas narrow band or white noise tinnitus remains unaffected by such stimulation $[7,10]$.

As burst firing is a more powerful activator of the cortex than tonic firing [11-14] it may be expected that burst rTMS is more powerful in suppressing abnormal activity perceived as tinnitus than tonic rTMS. Recently it has become possible to deliver stimulations that consist of bursts of magnetic impulses (burst rTMS) [15]. Non-clinical studies have shown that short bursts of high-frequency $(50 \mathrm{~Hz})$ magnetic impulses repeated at $5 \mathrm{~Hz}$ (the frequency of the theta rhythm in the EEG) produce a controllable, consistent, long-lasting and powerful effect on motor $[15,16]$ and visual cortex [17] by suppressing excitatory circuits [16].

In this paper we describe that burst rTMS at frequencies of the EEG theta $(5 \mathrm{~Hz})$, alpha $(10 \mathrm{~Hz})$ and beta $(20 \mathrm{~Hz})$ rhythms applied to the auditory cortex can temporarily suppress narrow band noise or white noise tinnitus. 


\section{Materials and Methods}

Forty-six patients with narrow band noise or white noise tinnitus, which is unilateral or predominantly perceived as coming from one side, are included in the study. All participants undergo both burst and tonic rTMS of the auditory cortex at two separate dates. All patients who experience a tinnitus suppression during either tonic or burst rTMS or both sessions also undergo a sham stimulation: the presence of a placebo effect is tested by placing the coil perpendicular to the auditory cortex at the frequencies that yield maximal tinnitus suppression rates both for tonic and burst rTMS. Of the participants, 14 (30,4 \%) demonstrated no placebo effect on both tonic and burst rTMS, $9(19,6 \%)$ did have a placebo suppression, and $23(50 \%)$ showed no suppressive response to either burst nor tonic rTMS. Only results from the 14 patients who were placebofree rTMS responders were analyzed ( 7 women, 7 men; mean age 51.6 years: 55,4 years for men, 47,7 years for women, range $40-72$ years). Since the rTMS machine generates a clicking sound on each magnetic pulse delivery, using only results from placebo negative patients prevents the possible influence of sound from the rTMS masking the tinnitus. The rTMS is performed as a part of a continuing clinical protocol for selection of candidates for implantation of permanent electrodes for electrical stimulation of the auditory cortex for treatment for tinnitus $[7,8]$ the multidisciplinary tinnitus clinic of the University Hospital of Antwerp, Belgium. All prospective participants undergo a complete audiological, ENT and neurological investigation to rule out possible treatable causes for their tinnitus. Tinnitus matching is performed by presenting sounds to the ear in which the tinnitus is not perceived, and both tinnitus pitch and tinnitus intensity (above hearing threshold) are matched to the perceived tinnitus. Technical investigations include MRI of the brain and posterior fossa, pure tone and speech audiometry, Auditory Brainstem Response (ABR) and tympanometry. Assessment of tinnitus loudness is analysed by Visual Analogue Scale (VAS) while tinnitus severity is analysed by the Tinnitus Questionnaire[18] (TQ). Tinnitus duration is also recorded. This study is approved by the ethical committee of the University Hospital Antwerp, Belgium.

rTMS is performed using a super rapid stimulator (Magstim Inc, Wales, UK) with the figure of eight coil placed over the auditory cortex contralateral to the tinnitus side in a way previously described[19].

Before the rTMS session, patients grade their tinnitus on a VAS. The motor threshold to rTMS is first determined by placing the coil over the motor cortex. The intensity of the magnetic stimulation is slowly increased until a clear contraction is observed in the contralateral thenar muscle. The coil is then moved to a location over the auditory cortex contralateral to the side to where the patients refer their tinnitus $(5-6 \mathrm{~cm}$ in above the entrance of auditory meatus on straight line to the vertex). With the intensity of the stimulation set at $90 \%$ of the motor threshold, the site of maximal tinnitus suppression is determined using $1 \mathrm{~Hz}$ stimulation. When tinnitus suppression is noted that patient is asked to estimate the decrease in tinnitus in percentage using the VAS. The procedure is repeated with stimulations at $5 \mathrm{~Hz}$ (= theta frequency), followed by $10 \mathrm{~Hz}$ (= alpha frequency) and $20 \mathrm{~Hz}$ (= beta frequency), each stimulation session consisting of 200 pulses. When tinnitus suppression is induced by rTMS the patient is asked to notify when the tinnitus has returned back to baseline, i.e. when the tinnitus intensity is back to its initial VAS before the next rTMS frequency is applied. Burst stimulation is performed in a similar fashion on a separate day. Bursts are presented at the same frequencies, with 3 or 5 pulses per burst. For each patient the tonic and burst rTMS frequency that yield maximal tinnitus suppression was compared.

\section{Statistical analysis}

Data were analysed with SPSS 13.0. Differences in narrow band noise / white noise tinnitus suppression (\% reduction of tinnitus perception) after burst and tonic rTMS stimulation were explored using a paired sampled $t$-test with rTMS stimulation as dependent variable. Tinnitus suppression was better in all patients with burst rTMS than tonic rTMS, therefore further analyses are done on burst data only. To assess differences in tinnitus suppression between genders, an independent sampled $t$-test was performed with burst rTMS stimulation as dependent variable and gender as grouping variable. To assess differences in distress caused by tinnitus depending on the side (left or right) an independent sampled $t$-test was performed with Tinnitus Questionnaire (TQ) score as dependent variable and tinnitus side as grouping variable. Pearson's correlations were performed to assess significant correlations between variables.

\section{Results}

In 5 patients, maximal tinnitus suppression is obtained with theta $(5 \mathrm{~Hz})$ burst stimulation, in 2 with alpha $(10 \mathrm{~Hz})$ burst, and 7 with beta $(20 \mathrm{~Hz})$ burst stimulation.

Burst rTMS suppresses tinnitus that is perceived as narrow band/white noise better than tonic rTMS, $t(13)=6.4, p<.000$ (Figure 1). Women experience greater suppression of their tinnitus with burst stimulation than men, $t(12)=2.9, p<.05$ (Figure 2). Furthermore left sided tinnitus is perceived as more distressing on the TQ than right sided tinnitus, $t(12)=3.2, \mathrm{p}<.01$ (Figure $3)$.

The lower the tinnitus pitch around which the narrow band tinnitus is centred the more effectively rTMS suppresses tinnitus $r=-0.65, p<0.05$, (Figure 4) and the lower the tinnitus intensity the better the tinnitus suppression $r=0.6, p<0.005$ (Figure 4). 


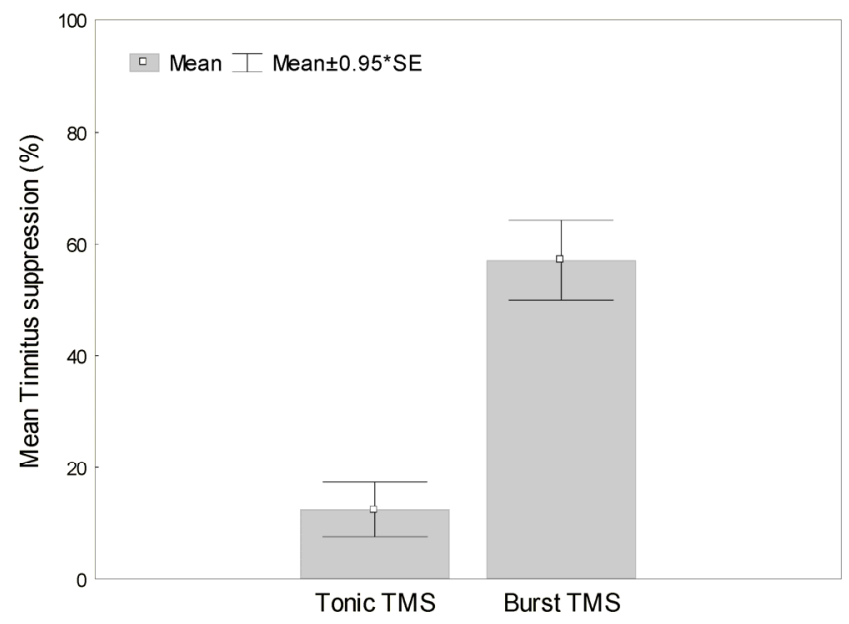

Figure 1: Mean tinnitus suppression (\%) with tonic and burst rTMS stimulation

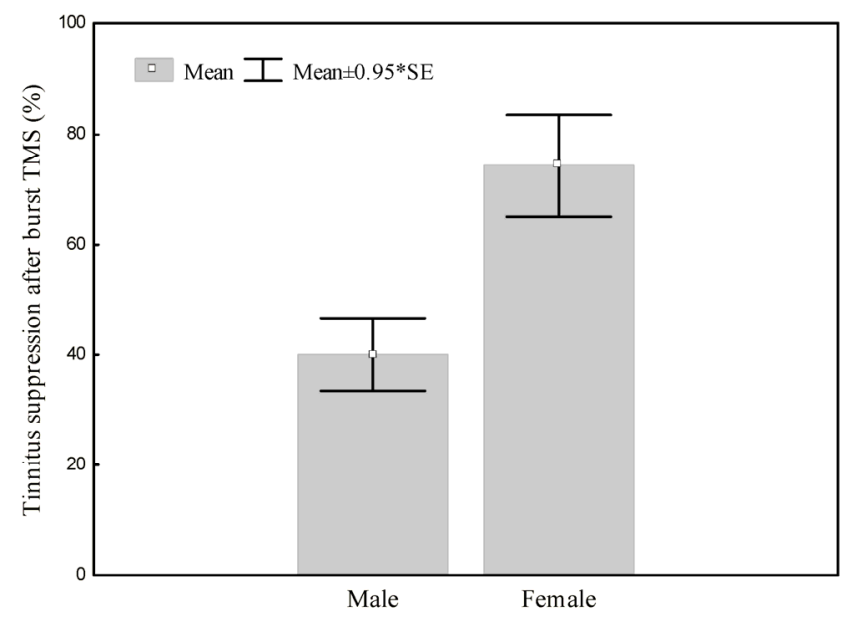

Figure 2: Mean tinnitus suppression with burst rTMS (\%) for male and female

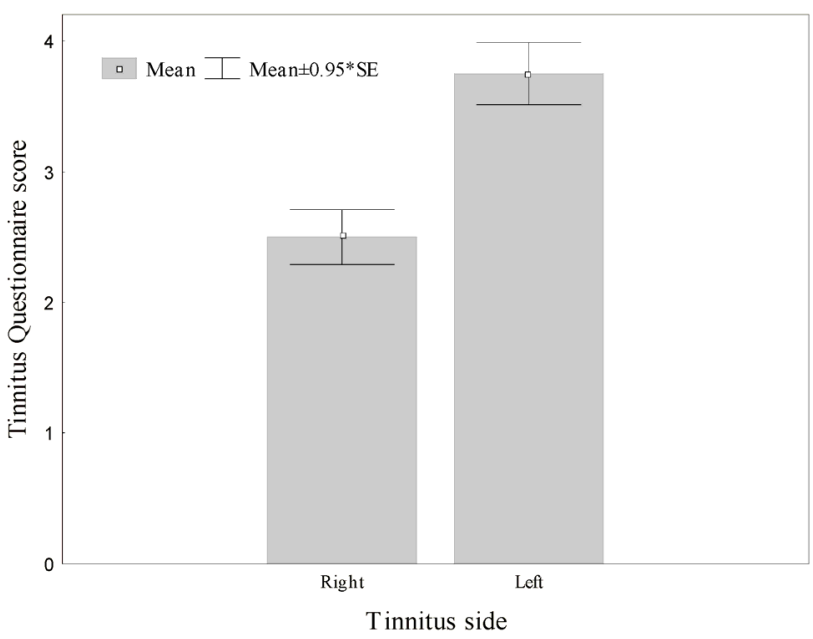

Figure 3: Mean Right and Left tinnitus distress measured on the Tinnitus Questionnaire
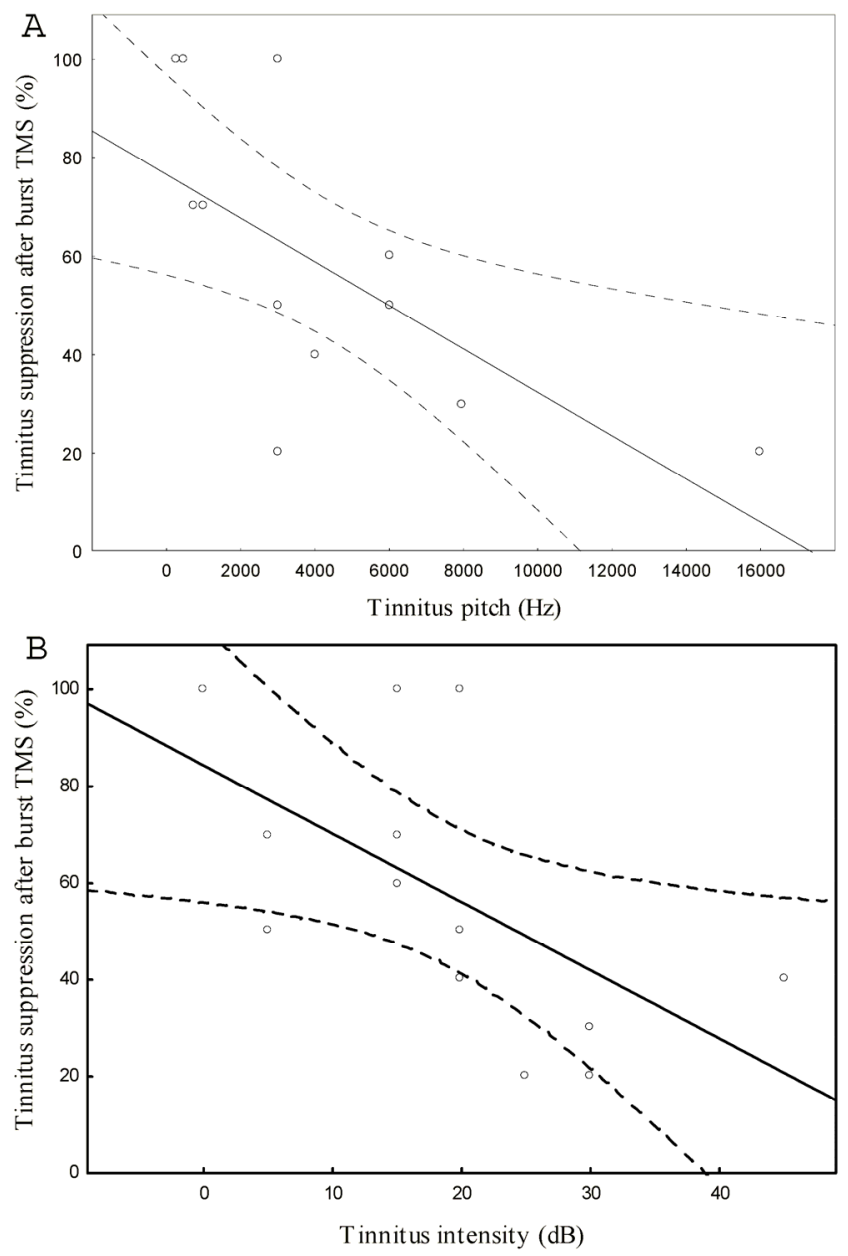

Figure 4: Mean tinnitus suppression after burst rTMS (\%) compared to (a) tinnitus pitch $(\mathrm{Hz})$ and (b) tinnitus intensity (dB)

\section{Discussion}

The present findings show that burst rTMS is effective in suppressing narrow band noise/white noise tinnitus. Our data demonstrate that it is not possible to suppress this kind of tinnitus by tonic rTMS. This is in accordance with electrical auditory cortex stimulation data [10] that have shown that it is not possible to suppress narrow band noise/white noise tinnitus with tonic electrical stimulation. The difference in the effect of single pulses and bursts on tinnitus may be explained by the fact that burst stimulation is more powerful in activating the cerebral cortex than tonic stimulation [11-14]. This may be related to the fact that burst activation requires less temporal integration to reach the threshold of a neuron and bursts may activate neurons that are not activated by tonic stimulations (unmasking dormant synapses) [2].

A second hypothetical explanation could be that only burst stimulation is capable of modifying the function of the extralemniscal (nonspecific or non-classical) system, the neurons of which are known to fire in bursts [20,21]. Tonic or single spike firing is the main firing mode of neurons in the tonotopically 
organized lemniscal auditory system [20-22]. Burst firing has been found to occur normally in neurons in the non-tonotopically organized extralemniscal auditory system [20-22]. Some cells in the ventral medial geniculate body (MGBv) of the thalamus can fire in both modes depending on their membrane potential $[23,24]$. In a depolarized state they fire in a tonic mode, in a hyperpolarized state they fire in burst mode. During alertness, neurons in the MGBv encode sound signals by firing action potentials in a tonic mode. When these neurons fire in burst, connections to other parts of the central nervous system may be opened through unmasking dormant synapses [2]. Externally applied burst stimuli may have the same effect.

There are indications that the extralemniscal auditory system is abnormally involved in tinnitus [25]. Furthermore, animal studies demonstrate that both tonic [26, 27] and burst [28, 29] firing are increased in the lemniscal and extralemniscal system respectively under conditions that are assumed to mimic tinnitus in humans. Therefore, increased activation of the burst-firing non-tonotopic extralemniscal pathways may lead to perception of tinnitus of noise character, whereas increased activation of the tonic firing tonotopic lemniscal system may produce pure tone tinnitus. The fact that bursts may activate neurons that are not activated by tonic stimulations [2] may explain why neural activity in the extralemniscal pathways cannot be suppressed by tonic stimulation but only by burst stimulation.

The second hypothesis is supported by the finding that patients who perceive their tinnitus having a low centered pitch respond better to burst stimulation than higher pitches. Neurons in the nuclei of the ascending auditory pathways that are tuned to low frequency sounds generally have wider tuning curves than neurons tuned to high frequency sounds. This means that auditory cortex cells processing low pitch sounds are less frequency specific than those processing high pitch sounds, and thus respond more like a non-tonotopic system in general. The tonotopic organization of Heschl's gyrus (primary auditory cortex) in humans demonstrates [4, 30-32] neural generators for low frequency sounds more laterally, and higher pitch sounds more medially. This could lead to the suggestions that low frequency tinnitus, generated more superficially, might be reached easier with rTMS than higher pitched tinnitus, which is presumably generated deeper in the Silvian valley. However, as the authors have expressed previously, based on fMRI and technical data [33], the influence of rTMS on the primary cortex is probably indirect, via modulation of the tonotopic map on the secondary auditory cortex [32], as there exist functional connections in humans between Heschl's gyrus and the lateral superior temporal gyrus [34].

To the authors knowledge this is the first report of burst rTMS using other frequencies than the well known theta burst [15-17]. In some patients only alpha or beta burst rTMS is capable of suppressing tinnitus, and theta burst not. Therefore in rTMS studies it could be worthwhile not to limit burst stimulation to theta burst rTMS.

The finding of this study that rTMS is more effective in suppressing the tinnitus of women thus show a similarity between rTMS treatment of tinnitus and that of microvascular decompression of the auditory nerve [35]. Finally we found that in this study left sided tinnitus is perceived as more distressing than right sided tinnitus. This is in accordance with published epidemiological data that show that people suffering left sided tinnitus complain more from tinnitus than people with right sided tinnitus [36].

\section{Conclusion}

Burst rTMS can be used clinically, not only theta burst, but also alpha and beta burst. Burst rTMS is capable of suppressing narrow band/white noise tinnitus very much better than tonic rTMS. This could be due the simple fact that burst neuromodulation is more powerful than tonic neuromodulation or to a differential effect of burst and tonic stimulation on the lemniscal and extralemniscal auditory system.

\section{Conflict of interest}

The authors have declared that no conflict of interest exists.

\section{References}

1. Jastreboff PJ. Phantom auditory perception (tinnitus): mechanisms of generation and perception. Neurosci Res 1990;8(4):221-54.

2. Moller A. Neural plasticity and disorders of the nervous system. Cambridge: Cambridge University Press, 2006.

3. Flor H, Elbert T, Knecht S, Wienbruch C, Pantev C, Birbaumer N, Larbig W, Taub E. Phantom-limb pain as a perceptual correlate of cortical reorganization following arm amputation. Nature 1995;375(6531):482-4.

4. Muhlnickel W, Elbert T, Taub E, Flor H. Reorganization of auditory cortex in tinnitus. Proc Natl Acad Sci U S A 1998;95(17):10340-3.

5. Salvi RJ, Wang J, Ding D. Auditory plasticity and hyperactivity following cochlear damage. Hear Res 2000;147(1-2):261-74.

6. Kaltenbach JA, Afman CE. Hyperactivity in the dorsal cochlear nucleus after intense sound exposure and its resemblance to tone-evoked activity: a physiological model for tinnitus. Hear Res 2000;140(1-2):165-72.

7. De Ridder D, De Mulder G, Verstraeten E, et al. Primary and secondary auditory cortex stimulation for intractable tinnitus. ORL J Otorhinolaryngol Relat Spec 2006;68(1):48-54.

8. De Ridder D, De Mulder G, Walsh V, Muggleton N, Sunaert S, Moller A. Magnetic and electrical stimulation of the auditory cortex for intractable tinnitus. Case report. J Neurosurg 2004;100(3):560-4.

9. Hallett M. Transcranial magnetic stimulation and the human brain. Nature 2000;406(6792):147-50.

10. De Ridder D, De Mulder G, Verstraeten E, et al. Primary and secondary auditory cortex stimulation for intractable tinnitus. ORL 2006; in press.

11. Lisman JE. Bursts as a unit of neural information: making unreliable synapses reliable. Trends Neurosci 1997;20(1):38-43.

12. Swadlow HA, Gusev AG. The impact of 'bursting' thalamic impulses at a neocortical synapse. Nat Neurosci 2001;4(4):402-8.

13. Sherman SM. Tonic and burst firing: dual modes of thalamocortical relay. Trends Neurosci 2001;24(2):122-6. 
14. Sherman SM. A wake-up call from the thalamus. Nat Neurosci 2001;4(4):344-6.

15. Huang $\mathrm{YZ}$, Edwards MJ, Rounis E, Bhatia KP, Rothwell JC. Theta burst stimulation of the human motor cortex. Neuron 2005;45(2):201-6.

16. Di Lazzaro V, Pilato F, Saturno E, et al. Theta-burst repetitive transcranial magnetic stimulation suppresses specific excitatory circuits in the human motor cortex. J Physiol 2005;565:945-50.

17. Franca M, Koch G, Mochizuki H, Huang YZ, Rothwell JC. Effects of theta burst stimulation protocols on phosphene threshold. Clin Neurophysiol 2006;117(8):1808-13.

18. Goebel G, Hiller W. [The tinnitus questionnaire. A standard instrument for grading the degree of tinnitus. Results of a multicenter study with the tinnitus questionnaire]. Hno 1994;42(3):166-72.

19. De Ridder D, Verstraeten E, Van der Kelen K, De Mulder G, Sunaert S, Verlooy J, Van de Heyning P, Moller A. Transcranial magnetic stimulation for tinnitus : influence of tinnitus duration on stimulation parameter choice and maximal tinnitus suppression. Otol Neurotol 2005;26(4):616-9.

20. He J, Hu B. Differential distribution of burst and single-spike responses in auditory thalamus. J Neurophysiol 2002;88(4):2152-6.

21. Hu B, Senatorov V, Mooney D. Lemniscal and non-lemniscal synaptic transmission in rat auditory thalamus. J Physiol 1994;479 ( Pt 2):217-31.

22. Hu B. Cellular basis of temporal synaptic signalling: an in vitro electrophysiological study in rat auditory thalamus. J Physiol 1995;483 ( Pt 1):167-82.

23. Schwarz DW, Tennigkeit F, Puil E. Metabotropic transmitter actions in auditory thalamus. Acta Otolaryngol 2000;120(2):251-4.

24. Tennigkeit F, Puil E, Schwarz DW. Firing modes and membrane properties in lemniscal auditory thalamus. Acta Otolaryngol 1997;117(2):254-7.

25. Moller AR, Moller MB, Yokota M. Some forms of tinnitus may involve the extralemniscal auditory pathway. Laryngoscope 1992;102(10):1165-71.

26. Jastreboff PJ, Brennan JF, Sasaki CT. An animal model for tinnitus. Laryngoscope 1988;98(3):280-6.

27. Komiya H, Eggermont JJ. Spontaneous firing activity of cortical neurons in adult cats with reorganized tonotopic map following pure-tone trauma. Acta Otolaryngol 2000;120(6):750-6.

28. Chen GD, Jastreboff PJ. Salicylate-induced abnormal activity in the inferior colliculus of rats. Hear Res 1995;82(2):158-78.

29. Eggermont JJ, Kenmochi M. Salicylate and quinine selectively increase spontaneous firing rates in secondary auditory cortex. Hear Res 1998;117(1-2):149-60.

30. Lutkenhoner B, Steinstrater O. High-precision neuromagnetic study of the functional organization of the human auditory cortex. Audiol Neurootol 1998;3(2-3):191-213.

31. Pantev C, Roberts LE, Elbert T, Ross B, Wienbruch C. Tonotopic organization of the sources of human auditory steady-state responses. Hear Res 1996;101(1-2):62-74.

32. Talavage TM, Ledden PJ, Benson RR, Rosen BR, Melcher JR. Frequency-dependent responses exhibited by multiple regions in human auditory cortex. Hear Res 2000;150(1-2):225-44.

33. De Ridder D. Tinnitus and auditory cortex :answer to a letter to the editor. J Neurosurg 2004;101(1):172-2.

34. Brugge JF, Volkov IO, Garell PC, Reale RA, Howard MA. Functional connections between auditory cortex on Heschl's gyrus and on the lateral superior temporal gyrus in humans. J Neurophysiol 2003;90(6):3750-63.

35. Moller MB, Moller AR, Jannetta PJ, Jho HD. Vascular decompression surgery for severe tinnitus: selection criteria and results. Laryngoscope 1993;103:421-7.

36. Hallberg LR, Erlandsson SI. Tinnitus characteristics in tinnitus complainers and noncomplainers. Br J Audiol 1993;27(1):19-27. 